\title{
Creación, espacio y educación. Una relectura artística del entorno como fuente de aprendizaje experiencial
}

\section{Creation, space and education. An artistic rereading of the environment as a source of experiential learning}

\author{
Andrés Torres Carceller \\ Universidad de Barcelona \\ andrestorres@ub.edu
}

Recibido: 14/09/2020 Revisado: 18/10/2020

Aceptado: 09/12/2020 Publicado: 14/12/2020

\section{Resumen:}

Con una concepción del espacio como elemento clave para el aprendizaje, adoptamos una voluntad creativa en el habitar, estableciendo cuatro ejes conceptuales (refugio, tránsito, no lugar, encuentro) sobre los que investigar nuestra relación con el entorno mediante la Educación Artística a partir de cuatro talleres. Redescubrir el espacio con toda la potencialidad artística que comporta nos permite una mirada transversal enriquecedora. Crear espacios polivalentes y flexibles que rompan con la rutina, que potencien la imaginación, que promuevan activamente relaciones, comunicaciones y encuentros, representa un poderoso elemento educativo.

Sugerencias para citar este artículo:

Torres Carceller, Andrés (2020). Creación, espacio y educación. Una relectura artística del entorno como fuente de aprendizaje experiencial. Tercio Creciente (Monográfico extraordinario IV), (pp. 7183), https://dx.doi.org/10.17561/rtc.extra4.5753

TORRES CARCELLER, ANDRÉS. Creación, espacio y educación. Una relectura artística del entorno como fuente de aprendizaje experiencial. Tercio Creciente (Monográfico extraordinario IV), diciembre 2020, pp. 71-83, https://dx.doi.org/10.17561/rtc.extra4.5753 


\section{Abstract:}

With a conception of space as a key element for learning, we adopt a creative will in living, establishing four conceptual axes (refuge, transit, not place, meeting) on which to investigate our relationship with the environment through Art Education based on four workshops. Rediscovering the space with all the artistic potential it contains allows us an enriching transversal view. Creating versatile and flexible spaces that break with routine, that enhance the imagination, that actively promote relationships, communications and encounters, represents a powerful educational element.

\section{Palabras clave \\ Espacio, educación, procesos creativos, educación artística}

\section{Keywords}

Space, education, creative processes, artistic education

Sugerencias para citar este artículo:

Torres Carceller, Andrés (2020). Creación, espacio y educación. Una relectura artística del entorno como fuente de aprendizaje experiencial. Tercio Creciente (Monográfico extraordinario IV), (pp. 7183), https://dx.doi.org/10.17561/rtc.extra4.5753

TORRES CARCELLER, ANDRÉS. Creación, espacio y educación. Una relectura artística del entorno como fuente de aprendizaje experiencial. Tercio Creciente (Monográfico extraordinario IV), diciembre 2020, pp. 71-83, https://dx.doi.org/10.17561/rtc.extra4.5753 


\section{Introducción}

La concepción museística ha ido variando desde mediados del siglo pasado de mero contenedor para conservar y exponer obras a un lugar que favorece y organiza espacios de participación, convirtiéndose en centros de reflexión, donde el visitante puede no solo analizar de forma razonada el contenido expuesto, sino también abordarlo mediante una experimentación centrada en los sentidos y emociones. Con esta voluntad se organizó L'educació de les arts II. Els Ilocs de les arts: escola, transversalitat i xarxes que a través de una muestra, un ciclo de talleres y diversas conferencias, pretendía evidenciar cómo las artes pueden ser una pieza clave en el escenario emergente de innovación y transformación de la educación actual. Con el museo como campo de acción y mediante una metodología experiencial, un equipo de docentes de la facultad de educación diseñamos un ciclo de talleres y una instalación participativa que actuaba de nexo entre la muestra y los talleres.

Dirigidos a docentes de educación Infantil, Primaria y Secundaria, en ellos, abordamos el espacio desde cuatro metáforas diferentes: el refugio, el tránsito, el no lugar y el encuentro. Desarrollando en cada taller un itinerario de interacciones con el espacio que servía como detonante para mediante el lenguaje plástico, reflexionar en torno a cómo habitamos el espacio y a sus repercusiones educativas, además de incidir en el papel del arte en la escuela como medio de conocimiento.

\section{El espacio como elemento educativo}

El espacio es un elemento clave que dificulta o potencia la práctica educativa y no podemos limitarnos a considerar el espacio educativo simplemente como la organización del aula. El centro educativo, además de ser el lugar donde se desarrollan los procesos de enseñanza y aprendizaje, constituye a su vez un espacio habitado por personas con personalidades y necesidades diversas que lo viven de muchas maneras diferentes, sin que éste haya estado diseñado contemplando todas estas posibilidades. El rígido esquema que limita el espacio para una única función restringe tanto las posibilidades educativas como la propia calidad de vida de sus usuarios (Austin, 2009). 
Experiencias como las aulas abiertas, los rincones y ambientes de aprendizaje, la cocreación de espacios entre los diferentes agentes del centro, retoman el concepto reggio del espacio, aportando nuevas experiencias que rompen la concepción estática e inamovible del aula.

Habitar el espacio es más bien, un oxímoron. Habitar es, por definición, vivir, residir, estar. Acciones que tienen como escenario el hogar o la población. Habitar un espacio público como el museo, en cambio, nos remite a estos lugares de acceso común que, más que habitar, transitamos, atravesamos, ocupamos o colapsamos. Ante la imposibilidad física y conceptual de abarcar el mundo que lo rodea, el ser humano piensa en el lugar.

El umbral entre el dentro y el fuera, entre el entorno protector y el lugar que nos permite asomarnos al mundo, en los espacios que habitamos, se genera una dialéctica soterrada de relaciones y hábitos. Entendiendo los lugares no solo como escenario de nuestra existencia, sino como generadores de procesos creativos nos permite abordar el tema del espacio como elemento educativo desde un posicionamiento plenamente activo. Crear espacios polivalentes y flexibles que rompan con la rutina, que potencien la imaginación, que promuevan activamente relaciones, comunicaciones y encuentros, representa un poderoso elemento educativo. Promoviendo una relación del lugar y el tiempo para el desarrollo de una respuesta creativa, posibilitando a los docentes aprender libremente desde su propia experiencia, transformando el proceso de aprendizaje en una sucesión de descubrimientos que facilita la individualización de los conocimientos según diferentes intereses y sensibilidades. Involucrar a los participantes en el desarrollo de habilidades creativas complejas a partir de la experiencia sensorial del entorno, favorecer la integración de contenidos a través de elementos significativos de su entorno más próximo. A su vez, la puesta en común de los mismos, permite enriquecer los saberes del grupo obteniendo una visión global que multiplica las perspectivas sobre el tema y sus diferentes aplicaciones futuras, acentuando la motivación de los docentes por diseñar e implementar con sus alumnos propuestas didácticas innovadoras que integren el espacio como elemento vertebrador. La cohesión del grupo participante y la posibilidad de aprender entre iguales ha incrementado altamente su nivel de engagement, ideando de forma autónoma propuestas didácticas para explorar con sus alumnos nuevas formas de aprender. Proporcionando así un clima emocional que favorezca la autoestima y la confianza. Redescubrir el espacio con toda la potencialidad artística que comporta, nos permite una mirada transversal enriquecedora. 


\section{Una postura creativa del habitar}

Con la intención de afrontarlo más allá de simple continente, diseñamos una serie de propuestas creativas a partir de abordar desde diferentes ángulos nuestro entorno para reflexionar sobre el espacio y el arte en el aprendizaje, explorando éste como un medio generador de conocimiento. Estas propuestas, creadas por un equipo de docentes de la Facultad de Educación han sido diseñadas para la formación de docentes de las distintas etapas de la educación obligatoria, fomentando los procesos artísticos como medio de investigación.

El Arte también es una forma de conocimiento, por lo que emplear los procesos artísticos como medio de investigación, permite encarar los retos de maneras diferentes, desarrollando el pensamiento divergente. La investigación en el campo de la Educación Artística establece un nexo de unión entre el ámbito educativo y el artístico, que generalmente están disociados. Por lo que investigar en Educación Artística constituye un campo diverso que comprende con límites difusos múltiples disciplinas, permitiendo el desarrollo complementario de competencias aparentemente dispares.

Con la intención de fomentar el aprendizaje por la acción, no pretendíamos enseñar conceptos sino que mediante la práctica de procesos creativos generados a partir de los diferentes espacios del Centro de Arte Arts Santa Mònica, los docentes-alumnos construyan sus propias conclusiones y propuestas de futuro, para que una vez de vuelta a su rol de docentes, las implementaran con sus estudiantes.

Nuestro planteamiento de los talleres conecta directamente con el valor que Dewey (1949) otorga a la experiencia en sí como generadora de aprendizajes, suponiendo una transformación del sujeto por la relevancia de los conocimientos adquiridos de esta forma. La experiencia no divide a quien conoce de lo que conoce.

Enfatizando además, que las experiencias artísticas tienen un poder mayor de transformación debido a que suponen un cuestionamiento de los conocimientos adquiridos, debiendo reelaborarlos para darles una utilidad operacional que permita aunar los conocimientos teóricos y procedimentales para seleccionar mediante la intuición la mejor estrategia para alcanzar los fines deseados. 
Cualquier acción es impredecible en cuanto escapa de las intenciones iniciales a través de múltiples interacciones y variables (Torres, 2019). Por lo que el sujeto debe desarrollar la capacidad de reacción mediante el pensamiento creativo, generando sus propios aprendizajes a través del tanteo.

El docente se transforma pues en un facilitador que diseña los elementos y situaciones necesarios para que los educandos exploren libremente en ellos. Creando sinergias grupales de conocimiento, al compartir sus avances y descubrimientos.

La incertidumbre, la no linealidad, son constantes en los procesos artísticos y preparan al sujeto para desarrollar el pensamiento creativo. Porque las respuestas desde el Arte son plurales y codificadas desde un plano personal que debe ser interpretado. En este sentido, "el arte es una forma de conocimiento basado en el principio de comunicabilidad de complejidades no necesariamente inteligibles" (Wagensberg, 2003, p.110).

Los procesos complejos de pensamiento que posibilitan las actividades creativas, permiten un enfoque transdisciplinar que potencia los aprendizajes significativos y contextualizados (Guillaumín, 2009). Bajo este pretexto, afrontamos la exploración cognitiva del espacio, poniendo en juego los sentimientos y los sentidos para alimentar a partir de la propia experiencia el germen creativo, que posteriormente se desarrolla de forma individual o en grupo entrelazándose en diferentes actividades secuenciadas que forman un recorrido para profundizar desde el tanteo la esencia del espacio como generador de conocimiento. Porque la creatividad contribuye tanto al desarrollo positivo de la personalidad y el pensamiento abstracto como a adquirir habilidades necesarias para resolver situaciones y retos. La experimentación libre y sensorial en cada sesión, por otra parte, permite activar la habilidad para resolver problemas, para razonar, para estimular el pensamiento crítico y creativo, desarrollando la capacidad para plantearnos cuestiones sobre lo que percibimos para encontrar nuestras propias respuestas. Así pues, el aprendizaje significativo desarrollado en este contexto de descubrimiento espacial nos ayuda a entender y a dar sentido a todo lo que nos rodea.

En base a la propuesta triangular de Ana Mae Barbosa (1991), para construir conocimientos en Arte (Contextualización histórica, práctica artística y lectura artística), adaptamos una secuencia para las diferentes actividades que se desarrollaban en los talleres: 
DOI: https://dx.doi.org/10.17561/rtc.extra4.5753 Investigación
Lugares y No Lugares para la creación Diciembre 2020

Procesos artísticos

Experiencia

Contextualización

Realización

Reflexión

El ciclo de talleres se dividía en cuatro sesiones aparentemente independientes pero entrelazadas entre sí, a partir de cuatro metáforas para aproximarse al espacio: refugio, tránsito, no-lugar, encuentro. De esta manera cada taller posibilita centrarse en una temática específica (Vicci, 2013) a través de diferentes tareas que se realizan de manera consecutiva y dan continuidad al conjunto. Asimismo nos parecía importante que la formación estuviera abierta a docentes de diferentes etapas educativas, para ampliar perspectivas y obtener resultados más heterogéneos que nos permitieran corroborar la riqueza de la interpretación de cada uno de los participantes en base a su personalidad y perfil profesional, y que a su vez la experiencia permitiese reflejar la importancia de potenciar la participación de grupos diferenciados para producir interacciones más enriquecedoras (Coutts y Jokela, 2008).

El Arte también es una forma de conocimiento, por lo que emplear los procesos artísticos como medio de investigación permiten profundizar en el conocimiento desde procesos de pensamiento divergente. La investigación en el campo de la Educación Artística establece un nexo de unión entre dos ámbitos, el educativo y el artístico que generalmente están disociados. Por lo que investigar en Educación Artística constituye un espacio variado 
que comprende con límites difusos múltiples disciplinas (Marín, 2011). Los talleres adoptan la misma línea que plantea el proyecto $A / r / t$ /ography desarrollado por la Universidad de la Columbia Británica (Vancouver), planteando la necesidad de indagar a través de cualquier proceso creativo que implique un enfoque transversal que favorezca reunir en la figura del docente de educación plástica las figuras de artista, investigador y maestro (Kalin, 2014). May, O’Donoghue e Irwin (2014) estudian la relación entre arte, aprendizaje y enseñanza analizan a partir de una experiencia de residencia entre artistas, pedagogos, docentes e investigadores, estableciendo la importancia de potenciar la capacidad de "aprender a aprender" de los docentes, y pese a la dificultad, intentar comprometerse en inspirarse en las formas y prácticas del arte contemporáneo.

Por otra parte, el espacio ya ha sido motor de múltiples propuestas didácticas desarrolladas desde la educación artística. Molina y Garrido (2008) con un marcado perfil social, establecieron una relación entre el espacio público y el exterior de una escuela infantil para facilitar la identificación con el lugar y transformar un paisaje para integrar aspectos de la identidad de sus habitantes. La óptica reconstruccionista (Neperud, 1995) que aplican en Finlandia para la formación de docentes, relacionado el Arte con la comunidad, vinculando ésta última con la tradición y el patrimonio cultural, entendiendo la comunidad como lugar, relacionando la educación -y el arte- con la vida (Villeneuve y Sheppard, 2009).

\section{Ciclo de talleres}

El formato escogido para desarrollar esta propuesta fue el de taller. Una forma organizativa tradicional, que nos permitía desplegar una serie de actividades en un espacio concreto durante un periodo de tiempo determinado suficientemente largo como para permitir conseguir provocar una secuencia didáctica completa y exhaustiva. La secuencia se ideó para favorecer un aprendizaje significativo que permitiera contextualizar la práctica realizada, experimentando en primera persona, reflexionando sobre la experiencia y analizando referentes artísticos, participando de un proceso creativo completo que permitiese interpretar la propia creación como cierre final. 
Nuestro enfoque pretendía abordar el espacio desde los microentornos, es decir, el marco inmediato donde nos situamos como personas. Pero sin obviar tampoco los macroentornos porque tampoco pretendíamos un trabajo aislado, separado de la realidad de cada individuo, negando que el contexto social, cultural e institucional afectan directa o indirectamente nuestras acciones. Pero desde nuestro interés por dirigir nuestra atención hacia lo cercando, ya que el macroentorno escapa a nuestra capacidad de influencia, pretendíamos transformar nuestro entorno inmediato para potenciar la creatividad, convirtiéndolo en el eje de trabajo, creando un contexto práctico que favorezca investigar el espacio cognitivamente, dando una mayor importancia a los sentidos y los sentimientos. Convirtiendo el desarrollo de los talleres en un ecosistema creativo donde se suman el biotipo educativo (arquitectura) y la biocenosis educativa (docentes).

Este ecosistema creativo debía permitir, además, romper la vinculación de lo inmediato y lo mediato, entre la cercanía y la distancia para facilitar esa presencia liberadora.

El acercamiento nos permitía generar una apertura de conocimiento al enfrentarnos a un mismo espacio desde un punto de vista siempre personal y diferente que lo convierte en único, y que permite una amplificación de la experiencia en total libertad, que buscaba liberarse de posibles ataduras e ideas preconcebidas.

El equipo creativo que diseñó la propuesta estaba formado por cuatro docentes: dos dirigían la acción, planteando las dinámicas a seguir y el ritmo de las mismas, mientras otros dos actuaban como miembros de soporte, tomando notas de las reacciones y documentando todo el proceso a nivel gráfico para recoger evidencias del desarrollo y asegurar el correcto funcionamiento de las diferentes actividades planteadas.

Cada uno de los talleres que conforman el ciclo partía de la misma secuencia didáctica utilizada como eje vertebrador catalizador y se desplegaba a través de una distribución atractiva y diferencial de las actividades desarrolladas en cada taller con unos objetivos específicos según cada caso. Se pretendía, de esta manera, facilitar el aprendizaje en cuanto a conocimientos, habilidades y actitudes utilizando esta secuencia estructural y aplicando diferentes técnicas y recursos artísticos, buscando un aprendizaje interdisciplinario y global fácilmente replicable a diferentes contextos educativos, pero con un enfoque destinatario recomendado inicialmente. 
En el primer taller abordamos el espacio desde la perspectiva del refugio, de nuestra necesidad natural de guarecernos y sentirnos seguros, explorando el espacio con una mirada íntima y primigenia. La metáfora de la concha o el caparazón para definir los espacios de refugio resultó válida. Pero desde el enfoque más amplio que nos permite la ecología simbólica, podemos ampliar el análisis a los aspectos personales que definen nuestros hogares, estos rasgos y valores esenciales de la propia personalidad, favorecen que nos sintamos seguros en nuestro espacio bajando nuestro nivel de alerta. Esto es extrapolable a cualquier espacio que hemos dispuesto para reflejarnos, donde podemos olvidarnos del mundo exterior y centrarnos completamente en nuestro yo más íntimo y en nuestro trabajo. Esto conecta con la necesidad infantil de buscar un espacio, de refugio donde nos sintamos seguros, que nos permite descansar del agotador reto de la convivencia, dándonos un tiempo de introspección, para posteriormente reintegrarse nuevamente con el resto de la sociedad. Esta necesidad de conexión con uno mismo y la sensación de paz que eso conlleva se plantearon como motores creativos del primer taller.

El segundo taller, el tránsito, tenía un planteamiento dinámico que nos permitía indagar en el concepto de los espacios de paso, convirtiendo el propio hecho de transitar en un elemento de creación. En esta ocasión se utilizó como referente el procedimiento situacionista de la deriva, que Debord (1999) define como un comportamiento lúdicoconstructivo para abordar psicogeográficamente ambientes diversos de forma ininterrumpida, oponiéndose a las nociones tradicionales de paseo o viaje. Durante el desarrollo de los talleres se puso en práctica lo que Debord acuñaba como deriva estática, poniendo el ejemplo de una jornada completa sin salir de la estación de Saint Lazare, limitando nuestro campo de acción en este caso al Centre d'Art Santa Mònica. Entendiendo la deriva como una forma de estudio del territorio no sistemática, que permite analizar los diferentes ambientes desde una perspectiva no estática con las emociones como elemento amplificador. Lo cual permite trazar una analogía entre el transitar y el gesto como medio gráfico de expresión.

El tercer taller, el no-lugar, enlaza en su punto de partida con el procedimiento de la deriva, conectando en su planteamiento azaroso con las promenades surrealistas, donde con la ayuda de la metamorfosis que la nocturnidad brinda a la ciudad, buscaban la realidad oculta subyacente, más íntima, sensorial, perceptiva. Lo cual sumado a la idea de la ciudad como laberinto que plantea Baudelaire (1974) nos permitía alejarnos de una concepción lógica del espacio, partiendo del concepto de mapa como herramienta para codificar y traducir el 
territorio, mezclándolo con la psicogeografía situacionista, para establecer un paradójico mapa tangiblemente intangible, no de un lugar, sino de un espacio mental y conceptual que representa el no-lugar.

El último taller, dedicado al encuentro, pretendía recoger todo lo surgido a partir de los diferentes enfoques de descubrimiento y experimentación espacial, evidenciando los aprendizajes subconscientes adquiridos para reflexionar sobre la importancia del papel de la educación Visual y Plástica, profundizando en su relación con el espacio al tomarlo como punto de origen y de final. Constituyendo un ágora, en la cual los docentes podían compartir ideas a través del uso de metodologías activas como el role playing, que no deja de ser un proceso de cocreación basado en la espontaneidad o el pensamiento visual, el visual thinking, como medio de análisis, configurando los pensamientos mediante estructuras que ordenan los conceptos para facilitar y establecer vínculos entre ellos. Este último taller permitía, de esta manera, conectar todo el aprendizaje significativo acumulado a lo largo del camino para utilizarlo como material de empoderamiento capaz de permitirles superar miedos, reticencias o inseguridades, liberando creativamente la mente de dichas ataduras.

\section{Conclusiones}

Nuestro objetivo no era producir obras de arte, sino generar a través de los procesos artísticos experiencias autoformativas que permitieran afrontar el tema del espacio desde las propias necesidades de cada participante en los talleres. No pretendíamos ofrecer recetas ni actividades ya creadas, sino la posibilidad de vivir la experiencia de afrontar el espacio más allá del simple hecho de ser el lugar que habitamos, explorándolo como un medio generador de conocimientos. Tampoco buscábamos mostrar un paquete cerrado que pueda ser luego puesto en práctica en las aulas, sino expandir un enfoque creativo que permita generar nuevas propuestas didácticas para ser desarrolladas en diferentes etapas y contextos educativos. Potenciando la figura del docente imaginativo y autónomo, que no se conforma con repetir año tras año las mismas propuestas, sino que afronta la educación desde posicionamientos autocríticos que le llevan a investigar y reelaborar nuevas vías.

La experimentación espacial, genera un alto efecto motivador en los participantes y estimula la creatividad. Se potencia además la adquisición de nuevos aprendizajes y valores a través del trabajo común en el espacio, redescubriendo éste desde una nueva perspectiva 
creativa más sensorial e inspiradora a través de la experimentación libre. Esta experimentación, que ayudará posteriormente a ordenar las ideas trabajadas indirectamente de forma exploratoria, requiere necesariamente de una iniciativa personal, de la posibilidad de una toma de decisión activa y de un nivel de conocimiento de la realidad que nos rodea mucho más profundo que el que nos aporta la simple observación. Centrar nuestra atención en el espacio cotidiano, en el espacio más próximo, nos permite indagar nuestra forma de relacionarnos en y con él, aumentando nuestra consciencia sobre como este nos afecta y condiciona, explorando nuevas relaciones que se establecen automáticamente entre morador y continente.

Tras la realización de los talleres pudimos constatar un creciente nivel motivacional entre los participantes, al experimentar libremente en el espacio interactuando con él. Las actividades realizadas en cada uno de los talleres permitieron pasar de la interdependencia a la independencia en materia educativa, generando una sinergia creativa capaz de provocar cambios, participando en un proceso de crecimiento que afectaba su propio yo y se retroalimentaba al mismo tiempo al grupo. A través de la incentivación espacial como elemento educativo se consigue potenciar la relación entre las necesidades humanas y pedagógicas en lugar de limitarlos.

\section{Referencias}

Austin, R. (2009). Deja que el mundo exterior entre en el aula: nuevas formas de enseñar y aprender más allá del aula de educación infantil. Morata.

Barbosa, A. M. (1991). A imagen no ensino da arte. Perspectiva.

Baudelaire, C. (1974). Poesía completa. Río nuevo.

Coutts, G., y Jokela, T. (eds.) (2008). Art, Community and Environment: Educational Perspectives. Intellect books.

Debord, G. (1999). Teoría de la deriva. En Internacional Situacionista, 2 (50-53). Literatura Gris.

Dewey. J. (1949). El arte como experiencia. Fondo de Cultura Económica.

Guillaumín, A. (2009). Suite para otra educación universitaria. En Guillaumín, A.; Octavio, O. (ed.). Hacia una nueva educación. Miradas desde la complejidad (111-135). Arana. 
Kalin, N. M. (2014). A/r/t ography as indisciplinary turning. Visual Inquiry, 3 (2), 131-146. https://doi.org/10.1386/vi.3.2.131_1

Marín, R. (2011). La investigación en Educación Artística. Educatio Siglo XXI, 29 (1), 211-230.

May, H, O'Donoghue, D, y Irwin, R (2014). Performing an intervention in the space between art and education. International Journal of Education Through Art, 10 (2), 163177. https://doi.org/10.1386/eta.10.2.163 1

Molina, J. A., y Garrido, P. A. (2008). Escribir el Lugar: collaborative projects in public spaces. International Journal of Education Through Art, 4 (2), 195-206. https://doi.org/10.1386/eta.4.2.195_1

Neperud, R. (ed.) (1995). Context, Content and Community in Art Education. Virginia NAEA.

Sabadell, LI. (2012). Guía per co-crear a l'escola. Consell Nacional de Cultura i de les Arts y Generalitat de Catalunya.

Torres, A. (2019). Aprendizaje creativo y educación visual y plástica; las artes como canal idóneo para desarrollar la creatividad. Brazilian Journal of Development, 5(6), 70727090. doi: https://doi.org/10.34117/bjdv5n6-193

Vicci, G. (Coord.) (2013). Miradas nómadas. Educación artística y cultura visual en Paysandú. Universidad de la República.

Villeneuve, P., y Sheppard, D. (2009): Close to home; Studying art and your community. Art Education, 62, (1), 6-13. https://doi.org/10.1080/00043125.2009.11518998

Wagensberg, J. (2003). Ideas sobre la complejidad del mundo. Tusquets. 